\title{
LTR retrotransposons in green plants show multiple examples of convergent evolution
}

Biryukov M.*, Ustyantsev K.

Institute of Cytology and Genetics SB RAS, Novosibirsk, Russia

*e-mail: birykov@bionet.nsc.ru

Key words: LTR retrotransposons, convergent evolution, ribonuclease H, Tat

Motivation and Aim: An illustrative example of modular evolution is independent acquisition of additional ribonuclease $\mathrm{H}(\mathrm{aRNH})$ domains by distant evolutionary lineages of long terminal repeat retrotransposons (LTR-RTs) from genomes of green plants (Tat), parasitic protists oomycetes (Chronos and Archon), and vertebrate retroviruses. In all the cases, structures of the elements converged to a single 'retroviral' variant when the aRNH domain is fixed right after the original one. Tat LTR-RTs of flowering plants exhibit the highest structural diversity with various positions of aRNH in relation to the other domains. However, previous studies of Tat LTR-RTs diversity were mostly limited to flowering plants. At the moment there are numerous genome assemblies of non-flowering plants available which opens a great perspective to study early evolution of Tat LTR-RTs, and understand its general tendencies.

Methods and Algorithms: We implemented a standalone pipeline written in Python which uses NCBI RPSBLAST and CDD to mine LTR-RTs sequences with aRNH from genome assemblies of 72 non-flowering green plant genomes, automatically annotates domain structure of the identified elements, clusters them and returns non-redundant set of best-scored representative sequences for phylogenetic analysis. The phylogeny was evaluated using IQ-TREE.

Results: We analysed 72 genomes of non-flowering green plants. No aRNH-containing elements were found in genomes of green algae and liverworts. LTR-RTs with aRNH from ferns, lycophytes, mosses, and hornworts formed two new clusters outside Tat. Elements with the 'retroviral' structure were found in ferns, lycophytes, and hornworts. A new structural variant with aRNH upstream gag was identified in mosses and lycophytes. LTR-RTs with aRNH and the 'retroviral' structure from ancient gymnosperms (Ginkgo biloba, Gnetum montanum, and cypress species) formed a new cluster within Tat basal to Tat LTR-RTs of flowering plants. Phylogenetic analysis of aRNH suggests independent origin of aRNH domains in LTRRTs from (1) ferns and lycophytes, (2) hornworts, and (3) pinaceae species (Tat III), while (4) the aRNH domains of all other LTR-RTs found in non-flowering plants cluster together with aRNH from Tat LTRRTs of flowering plants.

Conclusion: With the data obtained from non-flowering plants, the diversity of aRNH-containing LTR-RTs is now spreads beyond Tat. Our analysis suggests at least 4 independent cases of plant LTR-RTs adapting the 'retroviral' structure, indicating its significance for the elements' selection. Structural convergence appears to be the leading tendency in evolution of plant LTR-RTs.

Acknowledgements: Supported by the RFBR (20-34-90114) and Russian State Budget Project No. 02592019-0010-C-01. 